ESAIM: COCV 19 (2013) 20-42

DOI: $10.1051 / \mathrm{cocv} / 2011196$
ESAIM: Control, Optimisation and Calculus of Variations

www.esaim-cocv.org

\title{
SINGLE INPUT CONTROLLABILITY OF A SIMPLIFIED FLUID-STRUCTURE INTERACTION MODEL
}

\author{
Yuning LiU ${ }^{1}$, TAKÉo TAKahashi ${ }^{1}$ And Marius TucsnaK ${ }^{1}$
}

\begin{abstract}
In this paper we study a controllability problem for a simplified one dimensional model for the motion of a rigid body in a viscous fluid. The control variable is the velocity of the fluid at one end. One of the novelties brought in with respect to the existing literature consists in the fact that we use a single scalar control. Moreover, we introduce a new methodology, which can be used for other nonlinear parabolic systems, independently of the techniques previously used for the linearized problem. This methodology is based on an abstract argument for the null controllability of parabolic equations in the presence of source terms and it avoids tackling linearized problems with time dependent coefficients.
\end{abstract}

Mathematics Subject Classification. 35L10, 65M60, 93B05, 93B40, 93D15.

Received March 25, 2011. Revised August 10, 2011.

Published online February 23, 2012.

\section{INTRODUCTION}

In this work we study the boundary null controllability of a simplified one dimensional model for the motion of a solid in a viscous fluid. More precisely, we consider the initial-boundary value problem

$$
\begin{cases}v_{t}(t, x)-v_{x x}(t, x)+v(t, x) v_{x}(t, x)=0 & (t \geqslant 0, \quad x \in[-1,1] \backslash\{h(t)\}), \\ v(t, h(t))=\dot{h}(t) & (t \geqslant 0), \\ M \ddot{h}(t)=\left[v_{x}\right](t, h(t)) & (t \geqslant 0), \\ v(t,-1)=u(t), \quad v(t, 1)=0 & (t \geqslant 0), \\ h(0)=h_{0}, & \dot{h}(0)=h_{1}, \\ v(0, x)=v_{0}(x) \quad x \in[-1,1] \backslash\left\{h_{0}\right\} . & \end{cases}
$$

In the above equations, $v$ stands for the velocity field of the fluid, $M$ is the mass of the solid, whereas $h$ denotes the trajectory of the mass point moving in the fluid. The system is controlled by imposing the velocity of the fluid at the left-end of the tube and the corresponding control function is denoted by $u(t)$. The symbol $[f](x)$ represents the jump of $f$ at $x$, i.e.

$$
[f](x)=f(x+)-f(x-)
$$

\footnotetext{
Keywords and phrases. Null-controllability, fluid-structure interaction, viscous Burgers equation.

1 Institut Elie Cartan, Nancy Université/CNRS/INRIA, BP 70239, 54506 Vandoeuvre-lès-Nancy, France.

liuyuning.math@gmail.com; takeo8@gmail.com; Marius.Tucsnak@iecn.u-nancy.fr
} 
where $f(x-)$ and $f(x+)$ denote the left and right limits of $f$ at $x$, respectively. We write $v_{t}, v_{x}, v_{x x}$ for the derivatives of $v$ with respect to $t, x$ and for the second derivative of $v$ with respect to $x$. If a function only depends on $t$, we just write $\dot{h}$ and $\ddot{h}$ for the first and second derivatives of $h$ with respect to $t$.

This simplified model has been introduced by Vázquez and Zuazua in [17], where the well-posedness (with $(-1,1)$ replaced by $\mathbb{R}$ ) has been considered (see also [16]). The null controllability with controls at both ends has been considered by Doubova and Fernández-Cara in [4]. In this work the authors use a linearized problem with variable time-dependent coefficients, which is tackled by using the global Carleman estimates for the heat equation (see Imanuvilov and Fursikov [6]). As stated in [4], Remark 1.2, this methodology does not allow to extend the obtained results to the case of a control acting at only one end, due to the lack of connectivity of the fluid domain. In this work we tackle the case of controls acting at only one end by using a quite different strategy, which does not appeal at any Carleman estimate. Firstly, our linearized problem is with constant coefficients, so it can be tackled by spectral calculations combined with a classical method due to Fattorini and Russell. Secondly, we introduce a new iterative algorithm for the null-controllability in presence of source terms. The proposed iterative method for null-controllability in presence of source terms is of independent interest and therefore it is presented in an abstract setting. Note that this iterative method uses an idea going back to Lebeau and Robbiano [10], which consists in controlling a part of the state trajectory and using the dissipative character of parabolic equations to steer the remaining part to zero (see also Miller [11], Tenenbaum and Tucsnak [13]). Finally, we use a fixed point method introduced in Imanuvilov [9] and also used in Imanuvilov and Takahashi [8].

We also mention that similar controllability questions for the "full model" (i.e., involving two or three dimensional Navier-Stokes equations coupled with Newton's laws for rigid bodies) have been investigated in Boulakia and Osses [2] and in [8].

The main result in this paper asserts that the system (1.1) is locally null controllable in a neighborhood of zero and it can be stated as follows.

Theorem 1.1. Let $\tau>0$. There exists $r>0$ such that for every $h_{0} \in(-1,1), h_{1} \in \mathbb{R}, v_{0} \in H_{0}^{1}(-1,1)$, $v_{0}\left(h_{0}\right)=h_{1}$, satisfying $\left|h_{0}\right|+\left|h_{1}\right|+\left\|v_{0}\right\|_{H_{0}^{1}(-1,1)} \leq r$, there exists $u \in C[0, \tau]$ such that the solution of $(1.1)$ satisfies

$$
v \in L^{2}\left([0, \tau], H^{2}((-1,1) \backslash\{h(t)\}) \cap C\left([0, \tau], H^{1}(-1,1)\right), \quad h \in C^{1}[0, \tau],\right.
$$

together with

$$
\dot{h}(\tau)=0, \quad v(\tau, \cdot)=0 \quad \text { and } \quad h(\tau)=0 .
$$

Remark 1.2. The above result can be extended to show that, given $\tau>0$, any initial state $\left[\begin{array}{l}v_{0} \\ h_{0} \\ h_{1}\end{array}\right]$ with small enough norm in $L^{2}(-1,1) \times \mathbb{R} \times \mathbb{R}$ can be steered to rest in time $\tau$. To prove this fact, we take the control $u$ equal to zero for $t \in[0, \tau / 2]$ and we note that, by classical results on parabolic equations, we have

$$
v(\tau / 2, \cdot) \in H_{0}^{1}(-1,1), \quad v(\tau / 2, h(\tau / 2))=\dot{h}(\tau / 2) .
$$

We can thus conclude by controlling the system for $t \geq \tau / 2$ (this fact is possible due to the above regularity property and to Thm. 1.1).

The outline of the remaining part of this work is the following. In Section 2 we first recall some known results on null-controllability of parabolic equations and then we introduce a new iterative method for null controllability in the presence of source terms. This method, whose precise statement is given in Proposition 2.3, gives a general iterative framework to tackle source terms and, when applied to a given PDE system, does not require the use of Carleman estimates. In Section 3 we prove the null internal controllability for an auxiliary linear system, associated to (1.1), by combining some spectral estimates with the results from Section 2. Finally, Section 4 is devoted to the proof of the main result. 


\section{Some BACKGROUND ON NULL-CONTROLLABILITY}

We begin this section by briefly recalling some basic facts on the null-controllability of systems with negative generator. We next give the main result in this section, which concerns the controllability of these systems in the presence of a source term.

Throughout this section, $X$ and $U$ are Hilbert spaces which are identified with their duals, $\mathbb{T}$ is a strongly continuous semigroup on $X$, with generator $A: \mathcal{D}(A) \rightarrow X$ and $B \in \mathcal{L}(U, X)$. The inner product and the norm in $X$ are simply denoted by $\langle\cdot, \cdot\rangle$ and $\|\cdot\|$, respectively. We first consider classical linear control systems of the form

$$
\left\{\begin{array}{l}
\dot{z}=A z+B u, \\
z(0)=z_{0} \in X .
\end{array}\right.
$$

Definition 2.1. For $\tau>0$, the pair $(A, B)$ is said null-controllable in time $\tau$ if for every $z_{0} \in X$ there exists $u \in L^{2}([0, \tau], U)$ such that the solution of $(2.1)$ satisfies $z(\tau)=0$.

This means that for every $z_{0} \in X$, the set

$$
\mathcal{C}_{\tau, z_{0}}:=\left\{u \in L^{2}([0, \tau], U) \mid z(\tau)=0\right\},
$$

is non empty. The quantity

$$
K(\tau):=\sup _{\left\|z_{0}\right\|=1} \inf _{u \in \mathcal{C}_{\tau, z_{0}}}\|u\|_{L^{2}([0, \tau], U)}
$$

is then called the control cost in time $\tau$. If the pair $(A, B)$ is null-controllable in any time $\tau>0$, then it is known (see, for instance, [7], Thm. 3.1) that

$$
K: \mathbb{R}^{+} \rightarrow \mathbb{R}^{+} \quad \text { is continuous, non-increasing and } \lim _{\tau \rightarrow 0^{+}} K(\tau)=+\infty .
$$

The above definition for the control cost implies that for every function $\gamma: \mathbb{R}^{+} \rightarrow \mathbb{R}^{+}$, with $K(t)<\gamma(t)$ for every $t>0$, and for every $\tau>0$, there exists a control driving the solution of (2.1) to rest in time $\tau$ such that

$$
\|u\|_{L^{2}([0, \tau], U)} \leq \gamma(\tau)\left\|z_{0}\right\| \quad\left(z_{0} \in X\right) .
$$

We first recall a result which essentially goes back to Fattorini and Russell [5]. However, in order to have the precise statement we need in this work and in order to make the control cost precise, we give a short proof below.

Proposition 2.2. Assume that $A$ is a negative operator, admitting an orthonormal basis of eigenvectors $\left(\varphi_{k}\right)_{k \geqslant 1}$ and with the corresponding decreasing sequence of eigenvalues $\left(-\lambda_{k}\right)_{k \geqslant 1}$ satisfying

$$
\begin{gathered}
\inf _{k \geqslant 0}\left(\lambda_{k+1}-\lambda_{k}\right)=s>0, \\
\lambda_{k}=r k^{2}+O(k) \quad(k \rightarrow \infty),
\end{gathered}
$$

for some $r>0$. Moreover, assume that $U$ is a separable Hilbert space and that there exists a constant $m>0$ such that the adjoint operator $B^{*}$ satisfies

$$
\left\|B^{*} \varphi_{k}\right\|_{U} \geqslant m \quad(k \geqslant 1) .
$$

Then the pair $(A, B)$ is null-controllable in any time $\tau>0$ and there exist positive constants $M$, $C$, depending only on $\left(\lambda_{k}\right)_{k \geq 1}$ and on $m$, such that the control cost $K$ satisfies

$$
K(\tau)<C \mathrm{e}^{\frac{M}{\tau}} \quad(\tau>0) .
$$


Proof. According to a classical result, which goes back to Dolecki and Russell [3] (see also Tucsnak and Weiss [15], Sect. 11.2), the pair $(A, B)$ is null-controllable in time $\tau$ at cost $K(\tau)$ iff $\left(A^{*}, B^{*}\right)$ is final state observable in time $\tau$ at the same cost, i.e., if for every $K>K(\tau)$ we have

$$
K^{2} \int_{0}^{\tau}\left\|B^{*} \mathbb{T}_{t}^{*} z_{0}\right\|_{U}^{2} \mathrm{~d} t \geqslant\left\|\mathbb{T}_{\tau}^{*} z_{0}\right\|^{2} \quad\left(z_{0} \in X\right)
$$

where $\mathbb{T}_{t}^{*}$ is the adjoint of $\mathbb{T}_{t}$, whose generator is $A^{*}$. Simple calculations show that

$$
B^{*} \mathbb{T}_{t}^{*} z_{0}=B^{*} \mathbb{T}_{t} z_{0}=\sum_{k \geqslant 1} \mathrm{e}^{-\lambda_{k} t}\left\langle z_{0}, \varphi_{k}\right\rangle B^{*} \varphi_{k}
$$

The above formula, combined with the fact that $U$ admits an orthonormal basis $\left(\psi_{l}\right)_{l \geqslant 1}$, implies that

$$
\int_{0}^{\tau}\left\|B^{*} \mathbb{T}_{t}^{*} z_{0}\right\|_{U}^{2} \mathrm{~d} t=\sum_{l \geqslant 1} \int_{0}^{\tau}\left|\sum_{k \geqslant 1} \mathrm{e}^{-\lambda_{k} t}\left\langle z_{0}, \varphi_{k}\right\rangle_{X}\left\langle B^{*} \varphi_{k}, \psi_{l}\right\rangle_{U}\right|^{2} \mathrm{~d} t \quad\left(\tau>0, z_{0} \in X\right) .
$$

On the other hand, it is known (see, for instance, Tenenbaum and Tucsnak [14], Cor. 3.6, and references therein) that, under the assumptions (2.4) and (2.5), there exist constants $M_{1}, M_{2}>0$ (depending only on $s$ and on $r$ ) such that

$$
M_{1} \mathrm{e}^{\frac{M_{2}}{\tau}} \int_{0}^{\tau}\left|\sum_{k \geqslant 1} a_{k} \mathrm{e}^{-\lambda_{k} t}\right|^{2} \mathrm{~d} t \geqslant \sum_{k \geqslant 1}\left|a_{k}\right|^{2} \mathrm{e}^{-2 \lambda_{k} \tau} \quad\left(\tau>0, \quad\left(a_{k}\right) \in l^{2}\right) .
$$

The above estimate combined with (2.8) implies that

$$
M_{1} \mathrm{e}^{\frac{M_{2}}{\tau}} \int_{0}^{\tau}\left\|B^{*} \mathbb{T}_{t}^{*} z_{0}\right\|_{U}^{2} \mathrm{~d} t \geqslant \sum_{l \geqslant 1} \sum_{k \geqslant 1} \mathrm{e}^{-2 \lambda_{k} \tau}\left|\left\langle z_{0}, \varphi_{k}\right\rangle_{X}\right|^{2}\left|\left\langle B^{*} \varphi_{k}, \psi_{l}\right\rangle_{U}\right|^{2} \quad\left(\tau>0, z_{0} \in X\right) .
$$

The last inequality, together with (2.6), gives

$$
M_{1} \mathrm{e}^{\frac{M_{2}}{\tau}} \int_{0}^{\tau}\left\|B^{*} \mathbb{T}_{t}^{*} z_{0}\right\|_{U}^{2} \mathrm{~d} t \geqslant m^{2}\left\|\mathbb{T}_{\tau}^{*} z_{0}\right\|^{2} \quad\left(z_{0} \in X\right),
$$

so that (2.7) holds with $K=\frac{\sqrt{M_{1}} \mathrm{e}^{\frac{M_{2}}{2 \tau}}}{m}$. This fact clearly implies the conclusion of the proposition.

Assume that $(A, B)$ is null controllable in any time $\tau>0$. In the remaining part of this section we study a null-controllability problem associated to $(A, B)$ in the presence of source terms, i.e., we consider the system

$$
\left\{\begin{array}{l}
\dot{z}=A z+B u+f \\
z(0)=z_{0}
\end{array}\right.
$$

where $f:[0, \infty) \rightarrow X$.

We show below, roughly speaking, that if $f$ vanishes, with a prescribed decay rate, for some $\tau>0$, then there exists a control $u$ such that the solution of (2.9) tends to zero at a similar rate when $t \rightarrow \tau$. To make this assertion precise, we need some notation.

Let $q>1$ be a constant, let $\gamma:(0, \infty) \rightarrow[0, \infty)$ be a continuous non increasing function with $\lim _{t \rightarrow 0} \gamma(t)=$ $+\infty$ and let $\tau>0$. Consider a continuous function $\rho_{\mathcal{F}}:[0, \tau] \rightarrow[0, \infty)$ with

$$
\rho_{\mathcal{F}} \text { non increasing and } \rho_{\mathcal{F}}(\tau)=0,
$$


such that the function $\rho_{0}:\left[\tau\left(1-\frac{1}{q^{2}}\right), \tau\right] \rightarrow[0, \infty)$ defined by

$$
\rho_{0}(t):=\rho_{\mathcal{F}}\left(q^{2}(t-\tau)+\tau\right) \gamma((q-1)(\tau-t)) \quad\left(t \in\left[\tau\left(1-\frac{1}{q^{2}}\right), \tau\right]\right),
$$

is non increasing and

$$
\rho_{0}(\tau)=0 .
$$

The fact that, for each $\gamma$ as above, such a function $\rho_{\mathcal{F}}$ exists is easy to check. Indeed, we can take, for instance,

$$
\rho_{\mathcal{F}}\left(q^{2}(t-\tau)+\tau\right)=(\gamma((q-1)(\tau-t)))^{-(1+p)} \quad\left(t \in\left[\tau\left(1-\frac{1}{q^{2}}\right), \tau\right]\right),
$$

with $p>0$. We associate to the functions $\rho_{\mathcal{F}}$ and $\rho_{0}$ the Hilbert spaces $\mathcal{F}, \mathcal{U}$ and $\mathcal{Z}$ defined by

$$
\begin{aligned}
& \mathcal{F}=\left\{\begin{array}{l|l}
f \in L^{2}([0, \tau], X) & \left.\frac{f}{\rho_{\mathcal{F}}} \in L^{2}([0, \tau], X)\right\}, \\
\mathcal{U} & =\left\{\begin{array}{l|l}
u \in L^{2}([0, \tau], U) & \left.\frac{u}{\rho_{0}} \in L^{2}([0, \tau], U)\right\},
\end{array}\right. \\
\mathcal{Z}=\left\{z \in L^{2}([0, \tau], X)\right. & \left.\frac{z}{\rho_{0}} \in L^{2}([0, \tau], X)\right\},
\end{array}\right.
\end{aligned}
$$

where $\rho_{0}$ is any extension of the function in (2.11) (also denoted by $\left.\rho_{0}\right)$, initially defined on $\left[\tau\left(1-\frac{1}{q^{2}}\right), \tau\right]$, to a function which is continuous and non increasing on $[0, \tau]$. We can take, for instance,

$$
\rho_{0}(t)=\rho_{0}\left(\tau\left(1-\frac{1}{q^{2}}\right)\right) \quad\left(t \in\left[0, \tau\left(1-\frac{1}{q^{2}}\right)\right]\right),
$$

but the choice of this extension plays no role in what follows.

The inner product in $\mathcal{F}$ is defined by

$$
\left\langle f_{1}, f_{2}\right\rangle_{\mathcal{F}}=\int_{0}^{\tau} \rho_{\mathcal{F}}^{-2}(t)\left\langle f_{1}(t), f_{2}(t)\right\rangle \mathrm{d} t
$$

and similar definitions are used for $\mathcal{U}$ and for $\mathcal{Z}$. The induced norms are denoted by $\|\cdot\|_{\mathcal{F}},\|\cdot\|_{\mathcal{Z}}$ and $\|\cdot\|_{\mathcal{U}}$, respectively.

We are now in a position to prove the main result in this section.

Proposition 2.3. Assume that the pair $(A, B)$ is null-controllable in any time $t>0$, with control cost $K(t)$. Let $\gamma:(0, \infty) \rightarrow[0, \infty)$ be a continuous non increasing function such that

$$
K(t)<\gamma(t) \quad(t>0) .
$$

Let $\tau>0$ and let $\rho_{\mathcal{F}}$ and $\rho_{0}$ be two functions satisfying $(2.10)-(2.12)$ and let $(\mathcal{F}, \mathcal{Z}, \mathcal{U})$ be the corresponding Hilbert spaces, defined according to (2.13). Then, for every $z_{0} \in X$ and $f \in \mathcal{F}$, there exists $u \in \mathcal{U}$ such that the solution of (2.9) satisfies $z \in \mathcal{Z}$. Furthermore, there exists a positive constant $C$, not depending on $f$ and on $z_{0}$, such that

$$
\left\|\frac{z}{\rho_{0}}\right\|_{C([0, \tau], X)}+\|u\|_{\mathcal{U}} \leq C\left(\|f\|_{\mathcal{F}}+\left\|z_{0}\right\|_{X}\right) .
$$

In particular, since $\rho_{0}$ is a continuous function satisfying $\rho_{0}(\tau)=0$, the above relation yields $z(\tau)=0$. 
Remark 2.4. Note that the statement of the above proposition depends on an arbitrary constant $q>1$. The choice of this constant is important in applications to PDE systems. For instance to obtain Theorem 1.1, we will assume that $q^{4}<2$.

Before proving the above proposition, we recall the following classical result (see, for instance, Lem. 3.3 and Thm. 3.1 of [1], p. 80).

Lemma 2.5. Assume that $A$ is negative and $f:[0, \infty) \rightarrow X$. Let $\tau_{1}, \tau_{2}>0$ and let $z$ be the solution of

$$
\left\{\begin{array}{l}
\dot{z}=A z+f \quad t \in\left(\tau_{1}, \tau_{2}\right), \\
z\left(\tau_{1}\right)=a \in X .
\end{array}\right.
$$

There exists a positive constant $C$, depending only on $A$, such that

$$
\|z\|_{C\left(\left[\tau_{1}, \tau_{2}\right], X\right)}^{2}+\left\|(-A)^{1 / 2} z\right\|_{L^{2}\left(\left[\tau_{1}, \tau_{2}\right], X\right)}^{2} \leq\|a\|_{X}^{2}+C\|f\|_{L^{2}\left(\left[\tau_{1}, \tau_{2}\right], X\right)}^{2} \quad\left(a \in X, \quad f \in L^{2}\left(\left[\tau_{1}, \tau_{2}\right], X\right)\right) .
$$

Furthermore, if $a \in D\left((-A)^{\frac{1}{2}}\right)$, then we have

$$
\begin{aligned}
\|z\|_{H^{1}\left(\left(\tau_{1}, \tau_{2}\right), X\right)}^{2}+\left\|(-A)^{\frac{1}{2}} z\right\|_{C\left(\left[\tau_{1}, \tau_{2}\right], X\right)}^{2}+\|A z\|_{L^{2}\left(\left[\tau_{1}, \tau_{2}\right], X\right)}^{2} \leq\|a\|_{D\left((-A)^{\frac{1}{2}}\right)}^{2}+C\|f\|_{L^{2}\left(\left[\tau_{1}, \tau_{2}\right], X\right)}^{2} & \\
& \left(a \in D\left((-A)^{\frac{1}{2}}\right), \quad f \in L^{2}\left(\left[\tau_{1}, \tau_{2}\right], X\right)\right) .
\end{aligned}
$$

Proof of Proposition 2.3. Consider the sequence

$$
T_{k}=\tau-\frac{\tau}{q^{k}} \quad(k \geqslant 0) .
$$

First of all, let us remark that in that case, (2.11) yields

$$
\rho_{0}\left(T_{k+2}\right)=\rho_{\mathcal{F}}\left(T_{k}\right) \gamma\left(T_{k+2}-T_{k+1}\right) .
$$

We define the sequence $\left\{a_{k}\right\}_{k \geqslant 0}$ by $a_{0}=z_{0}$ and

$$
a_{k+1}=z_{1}\left(T_{k+1}-\right) \quad(k \geqslant 0),
$$

where $z_{1}$ satisfies

$$
\left\{\begin{array}{l}
\dot{z}_{1}=A z_{1}+f \\
z_{1}\left(T_{k}+\right)=0,
\end{array} \quad t \in\left(T_{k}, T_{k+1}\right), \quad(k \geq 0) .\right.
$$

On the other hand, for each $k \geqslant 0$ we consider the control system

$$
\left\{\begin{array}{l}
\dot{z}_{2}=A z_{2}+B u_{k} \quad\left(t \in\left(T_{k}, T_{k+1}\right)\right), \quad(k \geqslant 0), \\
z_{2}\left(T_{k}+\right)=a_{k} \in X,
\end{array}\right.
$$

where $u_{k} \in L^{2}\left(\left[T_{k}, T_{k+1}\right], U\right)$ is such that

$$
\begin{gathered}
z_{2}\left(T_{k+1}-\right)=0 \quad(k \geqslant 0), \\
\left\|u_{k}\right\|_{L^{2}\left(\left[T_{k}, T_{k+1}\right], U\right)}^{2} \leqslant \gamma^{2}\left(T_{k+1}-T_{k}\right)\left\|a_{k}\right\|_{X}^{2} \quad(k \geqslant 0) .
\end{gathered}
$$

The fact that such a control $u_{k}$ exists for every $k \in \mathbb{N}$ follows from the null-controllability of $(A, B)$ with a cost satisfying (2.14). 
To estimate the solution $z_{1}$ of (2.19), we note that from Lemma 2.5 it follows that

$$
\left\|z_{1}\right\|_{C\left(\left[T_{k}, T_{k+1}\right] ; X\right)}^{2}+\left\|(-A)^{1 / 2} z_{1}\right\|_{L^{2}\left(\left[T_{k}, T_{k+1}\right], X\right)}^{2} \leq C\|f\|_{L^{2}\left(\left[T_{k}, T_{k+1}\right], X\right)}^{2} \quad(k \geq 0) .
$$

In particular, recalling (2.18), we have

$$
\left\|a_{k+1}\right\|_{X}^{2} \leq C\|f\|_{L^{2}\left(\left[T_{k}, T_{k+1}\right], X\right)}^{2} \quad(k \geqslant 0) .
$$

Combining $(2.20),(2.21)$ and the fact that $\rho_{\mathcal{F}}$ is not increasing, we obtain that, for every $k \geqslant 0$,

$$
\begin{aligned}
\left\|u_{k+1}\right\|_{L^{2}\left(\left[T_{k+1}, T_{k+2}\right], U\right)}^{2} & \leq \gamma^{2}\left(T_{k+2}-T_{k+1}\right)\left\|a_{k+1}\right\|_{X}^{2} \\
& \leq C \gamma^{2}\left((q-1) \frac{\tau}{q^{k+2}}\right) \rho_{\mathcal{F}}^{2}\left(T_{k}\right)\left\|\frac{f}{\rho_{\mathcal{F}}}\right\|_{L^{2}\left(\left[T_{k}, T_{k+1}\right], X\right)}^{2} .
\end{aligned}
$$

Recalling the definition of $\rho_{0}(t)$ in (2.11) and using (2.17), we obtain

$$
\left\|u_{k+1}\right\|_{L^{2}\left(\left[T_{k+1}, T_{k+2}\right], U\right)}^{2} \leqslant C \rho_{0}^{2}\left(T_{k+2}\right)\left\|\frac{f}{\rho_{\mathcal{F}}}\right\|_{L^{2}\left(\left[T_{k}, T_{k+1}\right], X\right)}^{2} \quad(k \geqslant 0) .
$$

Since $\rho_{0}$ is non-increasing, it follows that there exists a positive constant, still denoted by $C$, such that

$$
\left\|\frac{u_{k+1}}{\rho_{0}}\right\|_{L^{2}\left(\left[T_{k+1}, T_{k+2}\right], U\right)}^{2} \leq C\left\|\frac{f}{\rho_{\mathcal{F}}}\right\|_{L^{2}\left(\left[T_{k}, T_{k+1}\right], X\right)}^{2} \quad(k \geqslant 0) .
$$

We define the control $u$ by

$$
u:=\sum_{k=0}^{\infty} u_{k} \mathbf{1}_{\left[T_{k}, T_{k+1}\right)},
$$

where $\mathbf{1}_{I}$ is the characteristic function of the set $I$. Combining (2.22) with (2.20) (for $k=0$ ) implies that there exists a positive constant $C$ such that

$$
\left\|\frac{u}{\rho_{0}}\right\|_{L^{2}([0, \tau], U)} \leq C\left(\left\|\frac{f}{\rho_{\mathcal{F}}}\right\|_{L^{2}([0, \tau], X)}+\left\|z_{0}\right\|\right) \quad\left(z_{0} \in X, \quad f \in \mathcal{F}\right) .
$$

If we set $z:=z_{1}+z_{2}$ then, for every $k \geqslant 0$, we have

$$
\left\{\begin{array}{l}
\dot{z}=A z+B u_{k}+f \quad\left(t \in\left[T_{k}, T_{k+1}\right]\right) \\
z\left(T_{k}\right)=a_{k}
\end{array}\right.
$$

Moreover,

$$
z\left(T_{k}-\right)=z_{1}\left(T_{k}-\right)+z_{2}\left(T_{k}-\right)=a_{k}=z_{1}\left(T_{k}+\right)+z_{2}\left(T_{k}+\right)=z\left(T_{k}+\right) \quad(k \geq 1),
$$

so that $z$ is continuous at $T_{k}$ for every $k \geqslant 0$. Consequently, $z$ satisfies $(2.9)$ for $t \in[0, \tau]$.

Furthermore, applying Lemma 2.5 to (2.24), we obtain the existence of a positive constant $C$ (depending only on $A$ and $B$ ) such that

$$
\|z\|_{C\left(\left[T_{k}, T_{k+1}\right], X\right)}^{2} \leq\left\|a_{k}\right\|_{X}^{2}+C\|u\|_{L^{2}\left(\left[T_{k}, T_{k+1}\right], X\right)}^{2}+C\|f\|_{L^{2}\left(\left[T_{k}, T_{k+1}\right], X\right)}^{2} \quad(k \geqslant 0) .
$$

The above estimate, combined with (2.20) imply that

$$
\|z\|_{C\left(\left[T_{k}, T_{k+1}\right], X\right)}^{2} \leq\left\|a_{k}\right\|_{X}^{2}+C \gamma^{2}\left(T_{k+1}-T_{k}\right)\left\|a_{k}\right\|_{X}^{2}+C\|f\|_{L^{2}\left(\left[T_{k}, T_{k+1}\right], X\right)}^{2} \quad(k \geqslant 0) .
$$


The above inequality and (2.21) imply that there exists a positive constant $C$, depending only on $A$ and $B$, such that

$$
\|z\|_{C\left(\left[T_{k}, T_{k+1}\right], X\right)}^{2} \leq C \gamma^{2}\left(T_{k+1}-T_{k}\right)\|f\|_{L^{2}\left(\left[T_{k-1}, T_{k+1}\right], X\right)}^{2} \quad(k \geqslant 1),
$$

and thus, since $\rho_{\mathcal{F}}$ is not increasing (recall also $(2.17)$ ),

$$
\begin{aligned}
\|z\|_{C\left(\left[T_{k}, T_{k+1}\right], X\right)}^{2} & \leq C \gamma^{2}\left(T_{k+1}-T_{k}\right) \rho_{\mathcal{F}}^{2}\left(T_{k-1}\right)\left\|\frac{f}{\rho_{\mathcal{F}}}\right\|_{L^{2}\left(\left[T_{k-1}, T_{k+1}\right], X\right)}^{2} \\
& =C \rho_{0}^{2}\left(T_{k+1}\right)\left\|\frac{f}{\rho_{\mathcal{F}}}\right\|_{L^{2}\left(\left[T_{k-1}, T_{k+1}\right], X\right)}^{2} \quad(k \geqslant 1) .
\end{aligned}
$$

Since $\rho_{0}$ is not increasing, we deduce from $(2.26)$ that

$$
\left\|\frac{z}{\rho_{0}}(t)\right\| \leqslant C\left\|\frac{f}{\rho_{\mathcal{F}}}\right\|_{L^{2}\left(\left[T_{k-1}, T_{k+1}\right], X\right)} \quad\left(k \geqslant 1, \quad t \in\left[T_{k}, T_{k+1}\right]\right) .
$$

The above estimate, combined with (2.20) and (2.25) (both for $k=0$ ), implies that

$$
\left\|\frac{z}{\rho_{0}}\right\|_{C([0, \tau], X)} \leq C\left(\left\|\frac{f}{\rho_{\mathcal{F}}}\right\|_{L^{2}([0, \tau], X)}+\left\|z_{0}\right\|_{X}\right) .
$$

The above estimate and (2.23) imply the conclusion (2.15).

Consider the backwards system

$$
\left\{\begin{array}{l}
-\dot{\zeta}(t)=A \zeta(t)+g \quad(t \in(0, \tau)) \\
\zeta(\tau)=0
\end{array}\right.
$$

By a duality argument (see, for instance, Thm. 4.1 in [8]), we obtain the following consequence of Proposition 2.3.

Corollary 2.6. Under the assumptions and notation of Proposition 2.3, for every $g \in L^{2}([0, \tau], X)$ the solution of (2.28) satisfies:

$$
\|\zeta(0)\|^{2}+\int_{0}^{\tau}\left\|\rho_{\mathcal{F}} \zeta\right\|^{2} \mathrm{~d} t \leq C\left(\int_{0}^{\tau}\left\|\rho_{0} g\right\|^{2} \mathrm{~d} t+\int_{0}^{\tau}\left\|\rho_{0} B^{*} \zeta\right\|_{U}^{2} \mathrm{~d} t\right) .
$$

Remark 2.7. Looking to estimate (2.29), one can easily think to Carleman estimates, such as those obtained in a PDE context in $[8,9]$. However, the way in which we obtained (2.29) is very far from Carleman estimates based strategies. Indeed, our only assumption is the pair $(A, B)$ is null controllable, without needing any knowledge on the methodolgy used to prove this controllability. In the application to our PDE system this gives the full choice of the method used to prove the null controllability of the pair $(A, B)$. In our application it turns out that a spectral method gives results which seem better than those obtained via Carleman estimates (see the next section).

The next proposition gives more information on the regularity of the controlled trajectory obtained in Proposition 2.3 .

Proposition 2.8. Under the notation and assumptions in Proposition 2.3, assume that there exists a continuous not increasing function $\rho:[0, \tau] \rightarrow \mathbb{R}^{+}$satisfying $\rho(\tau)=0$ and the inequalities

$$
\rho_{0} \leq C \rho, \quad \rho_{\mathcal{F}} \leq C \rho, \quad|\dot{\rho}| \rho_{0} \leq C \rho^{2} \quad(t \in[0, \tau])
$$


for some positive constant which does not depend on $t$. Then for every $z_{0} \in \mathcal{D}\left((-A)^{\frac{1}{2}}\right)$ there exists $u \in \mathcal{U}$ such that the solution $z \in \mathcal{Z}$ of (2.9) satisfies

$$
\frac{z}{\rho} \in L^{2}([0, \tau], \mathcal{D}(A)) \cap H^{1}((0, \tau), X) \cap C\left([0, \tau], \mathcal{D}\left((-A)^{\frac{1}{2}}\right)\right) .
$$

Moreover, there exists a positive constant $C$, depending only on $A$ and on $B$, such that

$$
\left\|\frac{z}{\rho}\right\|_{C\left([0, \tau], \mathcal{D}\left((-A)^{1 / 2}\right)\right) \cap H^{1}((0, \tau), X) \cap L^{2}([0, \tau], \mathcal{D}(A))} \leq C\left(\left\|z_{0}\right\|_{\mathcal{D}\left((-A)^{1 / 2}\right)}+\|f\|_{\mathcal{F}}\right) .
$$

Proof. Let $u$ be the control constructed in the proof of Proposition 2.3 and let $z$ be the corresponding trajectory. Then $w:=\frac{z}{\rho}$ satisfies

$$
\dot{w}=A w+\frac{B u}{\rho}+\frac{f}{\rho}-\frac{\dot{\rho} \rho_{0}}{\rho^{2}} \frac{z}{\rho_{0}} .
$$

Conditions (2.30) imply

$$
\frac{B u}{\rho}+\frac{f}{\rho}-\frac{\dot{\rho} \rho_{0}}{\rho^{2}} \frac{z}{\rho_{0}} \in L^{2}([0, \tau], X) .
$$

so that the conclusion easily follows by applying Lemma 2.5 .

In the remaining part of this section we consider a system obtained from (2.9) by adding a simple integrator. More precisely, we consider the equations

$$
\left\{\begin{array}{l}
\dot{z}=A z+B u+f, \\
\dot{h}=C z \\
z(0)=z_{0} \\
h(0)=h_{0}
\end{array}\right.
$$

where $Y$ is a Hilbert space and $C \in \mathcal{L}(X, Y)$. Consider the adjoint system of (2.33)

$$
\left\{\begin{array}{l}
-\dot{\xi}(t)=A \xi(t)+g(t)+C^{*} r, \\
\xi(\tau)=0
\end{array}\right.
$$

where $g:[0, \infty) \rightarrow X$ and $r \in Y$. The last result in this section is a characterization by duality of the fact that the solutions of (2.33) can be steered to rest at a prescribed decay rate. Using the notation introduced in (2.11)-(2.13), this result reads as follows.

Proposition 2.9. The following two statements are equivalent

(1) For every $(g, r) \in L^{2}([0, \tau], X) \times Y$, the solution of (2.34) satisfies

$$
\|r\|_{Y}^{2}+\|\xi(0)\|^{2}+\int_{0}^{\tau}\left\|\rho_{\mathcal{F}} \xi\right\|^{2} \mathrm{~d} t \leq C\left(\int_{0}^{\tau}\left\|\rho_{0} g\right\|^{2} \mathrm{~d} t+\int_{0}^{\tau}\left\|\rho_{0} B^{*} \xi\right\|_{U}^{2} \mathrm{~d} t\right) ;
$$

(2) there exists a bounded linear operator $E_{\tau}: X \times Y \times \mathcal{F} \rightarrow \mathcal{U}$ such that for any $\left(z_{0}, h_{0}, f\right) \in X \times Y \times \mathcal{F}$, the control $u=E_{\tau}\left(z_{0}, h_{0}, f\right)$ is such that the solution of (2.33) satisfies $z \in \mathcal{Z}$ and $h(\tau)=0$.

For the proof of this proposition, we refer to [8], Theorem 4.1. 


\section{InTERNAL NUlL CONTROLlABILITy FOR A LINEAR COUPLED PROBLEM}

In this section, we apply the result from Section 2 to obtain the internal null controllability of a linear system coupling the heat equation with some simple ODE's. This result will be used as a first step in the proof of Theorem 1.1. More precisely, we begin by studying a "fictitious" internal controllability problem in order to be able to apply the techniques in Section 2. Since, in the proof of Theorem 1.1, we will just need the spatial domain in which the heat equation holds to be of the form $(c, 0) \cup(0,1)$ with $c<-1$, we choose $c$ here to our best convenience, i.e., we take $c=-2$. In this context, Propositions 3.1 and 3.2 show that this linear system satisfies the assumptions of Proposition 2.2 which allows to obtain its null-controllability (Prop. 3.3). Then in Proposition 3.4, we show that we can control this linear system when coupled with a simple integrator (which corresponds to the position of the particle here) as in system (2.33). The last result, given in Corollary 3.5, gives some regularity properties of the controlled solution obtained in Proposition 3.4.

Denote

$$
X=L^{2}(-2,1) \times \mathbb{R},
$$

and consider the operator $A: \mathcal{D}(A) \rightarrow X$ defined by

$$
\begin{gathered}
\mathcal{D}(A)=\left\{z=\left[\begin{array}{l}
\varphi \\
g
\end{array}\right] \in H_{0}^{1}(-2,1) \times \mathbb{R} \mid \varphi(0)=g, \varphi_{\left.\right|_{(-2,0)}} \in H^{2}(-2,0), \varphi_{\left.\right|_{(0,1)}} \in H^{2}(0,1)\right\}, \\
A\left[\begin{array}{l}
\varphi \\
g
\end{array}\right]=\left[\begin{array}{c}
\varphi_{x x} \\
M^{-1}\left[\varphi_{x}\right](0)
\end{array}\right] .
\end{gathered}
$$

In the above formula $\varphi_{x x}$ is not understood in the distribution sense but it is just the function in $L^{2}(-2,1)$ whose restrictions to $(-2,0)$ and to $(0,1)$ coincide with the second derivative of $\varphi$ on each of those intervals. The space $X$ is endowed with the inner product

$$
\left\langle z_{1}, z_{2}\right\rangle=\int_{-2}^{1} \varphi_{1}(x) \varphi_{2}(x) \mathrm{d} x+M g_{1} g_{2}, \quad \text { where } \quad z_{i}=\left[\begin{array}{c}
\varphi_{i} \\
g_{i}
\end{array}\right], i=1,2 .
$$

We simply denote by $\|\cdot\|$ the induced norm.

The proof of the following result is quite standard so that we omit it.

Proposition 3.1. The operator $A$ is negative in $X$ (this means that $A$ is self-adjoint and that there exists $m_{1}>0$ such that $\langle A z, z\rangle \leqslant-m_{1}\|z\|^{2}$ for every $\left.z \in \mathcal{D}(A)\right)$.

The last proposition implies, according to the Lumer-Phillips theorem, that $A$ generates a contraction semigroup on X. Furthermore, since $A$ obviously has compact resolvents, it follows that there exists an orthonormal basis $\left(\Phi_{k}\right)$ in $X$ formed by eigenvectors of $A$ such that the corresponding eigenvalues form a non-increasing sequence $\left(-\lambda_{k}\right)$ with $\lambda_{k} \rightarrow \infty$.

The result below gives more information about the eigenvectors and the eigenvalues of $A$. To give the precise statement we introduce the Hilbert space $U=L^{2}\left(-2,-\frac{3}{2}\right)$ and the operator $B \in \mathcal{L}(U, X)$ defined by

$$
B u=\left[\begin{array}{c}
\mathbb{1}_{\left(-2,-\frac{3}{2}\right)} u \\
0
\end{array}\right] \quad(u \in U),
$$

where $\mathbb{1}_{\left(-2,-\frac{3}{2}\right)}$ stands for characteristic function of the interval $\left(-2,-\frac{3}{2}\right)$. Note here, for later use, that the adjoint $B^{*}$ of $B$ is given by

$$
B^{*}\left[\begin{array}{l}
\varphi \\
r
\end{array}\right]=\left.\varphi\right|_{\left(-2,-\frac{3}{2}\right)} \quad\left[\begin{array}{l}
\varphi \\
r
\end{array}\right] \in X
$$


Proposition 3.2. The sequence $\left(-\lambda_{k}\right)_{k \geq 1}$ is regular (i.e., $\left.\inf _{k \geqslant 1}\left(\lambda_{k+1}-\lambda_{k}\right)>0\right)$ and we have

$$
\lambda_{k}=\frac{k^{2} \pi^{2}}{9}+O(k) \quad(k \rightarrow \infty) .
$$

Furthermore, there exists a positive constant $c_{1}$ such that

$$
c_{1} \leq\left\|B^{*} \Phi_{k}\right\|_{U} \quad(k \geqslant 1) .
$$

Proof. Since $A$ is negative, all its eigenvalues are negative numbers. Let $-\mu^{2}$, with $\mu>0$ be an eigenvalue of $A$. This means that there exists a vector $\left[\begin{array}{l}\varphi \\ r\end{array}\right] \in \mathcal{D}(A) \backslash\{0\}$ such that

$$
\left\{\begin{array}{l}
-\varphi_{x x}=\mu^{2} \varphi(x) \quad x \in(-2,0) \cup(0,1), \\
\varphi(-2)=\varphi(1)=0, \\
\varphi(0)=-\frac{1}{M \mu^{2}}\left[\varphi_{x}\right](0) .
\end{array}\right.
$$

From the first two equations in (3.7) we deduce that

$$
\varphi(x)=\left\{\begin{array}{l}
C \sin (\mu(2+x)), x \in(-2,0), \\
D \sin (\mu(1-x)), x \in(0,1)
\end{array}\right.
$$

with $C, D \in \mathbb{R}$ and $C^{2}+D^{2} \neq 0$. In order to use the continuity of $\varphi$ at $x=0$ and the last equation in (3.7), we distinguish two cases.

The first case corresponds to the situation in which $\varphi(0)=0$. In this case, by the continuity of $\varphi$ at $x=0$ we obtain

$$
C \sin 2 \mu=0, \quad D \sin \mu=0 .
$$

The above equation and the last equation in (3.7) yield

$$
C \cos 2 \mu=D \cos \mu \text {. }
$$

From the above formula and (3.9), it easily follows that

$$
C^{2}=D^{2}
$$

This, combined with the fact that $C^{2}+D^{2} \neq 0$ and with (3.9), indicates that we have a first family $\left(-\lambda_{m}^{(1)}\right)_{m \geqslant 1}$ of eigenvalues of $A$ given by

$$
\lambda_{m}^{(1)}=\left(\mu_{m}^{(1)}\right)^{2}=m^{2} \pi^{2} \quad\left(m \in \mathbb{N}^{*}\right) .
$$

The second case corresponds to the situation in which $\varphi(0) \neq 0$. In this case, we have

$$
\sin (2 \mu) \neq 0, \quad \sin \mu \neq 0,
$$

and the constants $C$ and $D$ in the expression (3.8) of the eigenvectors can be chosen such that

$$
D=C \frac{\sin 2 \mu}{\sin \mu}=2 C \cos \mu .
$$

With this choice, the last condition in (3.7) yields the characteristic equation

$$
M \mu=\operatorname{cotan} \mu+\operatorname{cotan} 2 \mu .
$$


All the eigenvalues of $A$ not belonging to the family $\left(-\lambda_{m}^{(1)}\right)$, obtained in our first case, are of the form $\lambda=-\mu^{2}$, where $\mu$ is a positive root of (3.13). To locate the roots of this transcendental equation, note first that the expression in the right hand side of (3.13), suggests to study the function $f(\mu)=\operatorname{cotan} 2 \mu+\operatorname{cotan} \mu$, which is defined on the union of all intervals of the form $\left((m-1) \pi,(m-1) \pi+\frac{\pi}{2}\right)$ and $\left((m-1) \pi+\frac{\pi}{2}, m \pi\right)$, with $m \in \mathbb{N}^{*}$. Moreover, $f$ is decreasing on each of these intervals and

$$
\begin{gathered}
\lim _{\substack{\mu \rightarrow(m-1) \pi \\
\mu>(m-1) \pi}} f(\mu)=+\infty, \quad f\left((m-1) \pi+\frac{\pi}{3}\right)=0, \quad \lim _{\substack{\mu \rightarrow(m-1) \pi+\pi / 2 \\
\mu<(m-1) \pi+\pi / 2}} f(\mu)=-\infty, \\
\lim _{\substack{\mu \rightarrow(m-1) \pi+\frac{\pi}{\pi} \\
\mu>(m-1) \pi+\frac{\pi}{2}}} f(\mu)=+\infty, \quad f\left((m-1) \pi+\frac{2 \pi}{3}\right)=0, \quad \lim _{\substack{\mu \rightarrow m \pi \\
\mu<m \pi}} f(\mu)=-\infty .
\end{gathered}
$$

Therefore, we have two other families of eigenvalues of $A$, denoted $\left(-\lambda_{m}^{(2)}\right)_{m \geqslant 1}$ and $\left(-\lambda_{m}^{(3)}\right)_{m \geqslant 1}$ where

$$
\lambda_{m}^{(j)}=\left(\mu_{m}^{(j)}\right)^{2} \quad\left(j \in\{2,3\}, m \in \mathbb{N}^{*}\right)
$$

and $\mu_{m}^{(2)}, \mu_{m}^{(3)}$ can be written

$$
\mu_{m}^{(2)}=\frac{\pi}{2}+(m-1) \pi+\omega_{m}^{(2)} \quad\left(m \in \mathbb{N}^{*}\right)
$$

and

$$
\mu_{m}^{(3)}=(m-1) \pi+\omega_{m}^{(3)} \quad\left(m \in \mathbb{N}^{*}\right),
$$

where $\omega_{m}^{(2)} \in\left(0, \frac{\pi}{6}\right), \omega_{m}^{(3)} \in\left(0, \frac{\pi}{3}\right)$ and

$$
\lim _{m \rightarrow \infty} \omega_{m}^{(i)}=0, \quad i \in\{2,3\} .
$$

It is easily seen from (3.11), (3.14) and (3.15) that, for each $m \in \mathbb{N}^{*}$,

$$
\mu_{m}^{(3)}<\mu_{m}^{(2)}<\mu_{m}^{(1)}<\mu_{m+1}^{(3)}<\mu_{m+1}^{(2)}<\ldots,
$$

which implies that the sequences $\left(\mu_{m}^{(j)}\right)_{m \geqslant 1}$, with $j \in\{1,2,3\}$ have no elements in common. Furthermore, using that $\omega_{m}^{(2)} \in\left(0, \frac{\pi}{6}\right), \omega_{m}^{(3)} \in\left(0, \frac{\pi}{3}\right)$, we obtain that for each $m \in \mathbb{N}^{*}$ we have

$$
\begin{aligned}
& \mu_{m}^{(2)}-\mu_{m}^{(3)} \geq \frac{\pi}{2}+\omega_{m}^{(2)}-\omega_{m}^{(3)}>\frac{\pi}{6}, \\
& \mu_{m}^{(1)}-\mu_{m}^{(2)} \geq \frac{\pi}{2}-\omega_{m}^{(2)}>\frac{\pi}{3} \\
& \mu_{m+1}^{(3)}-\mu_{m}^{(1)} \geq \omega_{m+1}^{(3)} .
\end{aligned}
$$

In order to give a lower bound for $\omega_{m}^{(3)}$ we note that using (3.15), equation (3.13) writes

$$
\begin{aligned}
M\left((m-1) \pi+\omega_{m}^{(3)}\right)=\operatorname{cotan} & \left((m-1) \pi+\omega_{m}^{(3)}\right) \\
& +\operatorname{cotan}\left[2\left((m-1) \pi+\omega_{m}^{(3)}\right)\right]=\operatorname{cotan}\left(\omega_{m}^{(3)}\right)+\operatorname{cotan}\left(2 \omega_{m}^{(3)}\right) \quad\left(m \in \mathbb{N}^{*}\right) .
\end{aligned}
$$

Since

$$
\operatorname{cotan} x=\frac{1}{x}-\frac{1}{3} x+o\left(x^{2}\right) \quad(x \rightarrow 0),
$$

equation (3.19) writes

$$
M\left[(m-1) \pi+\omega_{m}^{(3)}\right]=\frac{3}{2 \omega_{m}^{(3)}}-\omega_{m}^{(3)}+o\left(\left(\omega_{m}^{(3)}\right)^{2}\right) .
$$


Multiplying both sides of the above formula by $\omega_{m}^{(3)} / M$ we get

$$
(m-1) \pi \omega_{m}^{(3)}=\frac{3}{2 M}+O\left(\left(\omega_{m}^{(3)}\right)^{2}\right) \quad(m \rightarrow \infty) .
$$

Dividing both sides of the above formula by $(m-1) \pi$ we obtain

$$
\omega_{m}^{(3)}=\frac{3}{2 \pi M(m-1)}+o\left(\frac{1}{m}\right) \quad(m \rightarrow \infty)
$$

which gives the desired lower bound for $\omega_{m}^{(3)}$.

Let now $\left(\mu_{m}\right)_{m \geq 1}$ denote the increasing sequence formed by rearranging all the values of the sequences $\left(\mu_{m}^{(i)}\right)_{m \geq 1}$, with $i \in\{1,2,3\}$. The the spectrum of $A$ is the set $-\Lambda$, where $\Lambda=\left(\lambda_{m}\right)_{m \geq 1}:=\left(\mu_{m}^{2}\right)_{m \geq 1}$. Combining (3.17), (3.18) and (3.22), we can see that $\Lambda$ is regular, which yields the first conclusion of our proposition.

We still have to show that $\Lambda$ satisfies (3.5). To achieve this goal denote

$$
\nu(K)=\max \left\{m \mid \mu_{m} \leqslant K\right\} \quad(K \geqslant 0) .
$$

It is clear that

$$
\nu(K)=\sum_{i=1}^{3} \nu^{(i)}(K) \quad(K \geqslant 0),
$$

where

$$
\nu^{(i)}(K)=\max \left\{m \mid \mu_{m}^{(i)} \leqslant K\right\} \quad(i \in\{1,2,3\}, \quad K \geqslant 0) .
$$

From (3.11), (3.14) and (3.15), it follows that

$$
\nu^{(i)}(K)=\frac{K}{\pi}+O(1) \quad(i \in\{1,2,3\}, \quad K \rightarrow \infty) .
$$

Combining (3.23) with (3.24), it follows that

$$
\nu(K)=\frac{3 K}{\pi}+O(1) \quad(K \rightarrow \infty) .
$$

Using the fact that

$$
\mu_{\nu(K)}=K+O(1) \quad(K \rightarrow \infty),
$$

setting $\nu(K)=m$ in the last formula and using (3.25) we obtain that

$$
\mu_{m}=\frac{m \pi}{3}+O(1) \quad(m \rightarrow \infty)
$$

which clearly implies (3.5).

We finally check estimate (3.6). For every $m \in \mathbb{N}^{*}$, the unitary eigenvector associated to the eigenvalue $\left(-\mu_{m}^{2}\right)$ is given by $\Phi_{m}=\left[\begin{array}{c}\varphi_{m} \\ \varphi_{m}(0)\end{array}\right]$ with

$$
\varphi_{m}(x)= \begin{cases}C_{m} \sin \left(\mu_{m}(2+x)\right), & x \in(-2,0), \\ D_{m} \sin \left(\mu_{m}(1-x)\right), & x \in(0,1),\end{cases}
$$

where, in order to ensure the normalization condition, we have

$$
C_{m}^{2}\left(1-\frac{\sin 4 \mu_{m}}{4 \mu_{m}}\right)+D_{m}^{2}\left(\frac{1}{2}-\frac{\sin 2 \mu_{m}}{4 \mu_{m}}\right)+M C_{m}^{2} \sin ^{2}\left(2 \mu_{m}\right)=1 .
$$


We deduce from (3.4) that

$$
B^{*} \Phi_{m}=\left.\varphi_{m}\right|_{\left(-2,-\frac{3}{2}\right)}
$$

so that

$$
\left\|B^{*} \Phi_{m}\right\|_{U}^{2}=\frac{C_{m}^{2}}{4}\left(1-\frac{\sin \mu_{m}}{\mu_{m}}\right)
$$

From (3.26), we deduce that there exists $d_{1}>0$ such that

$$
d_{1}<1-\frac{\sin \mu_{m}}{\mu_{m}} \quad\left(m \in \mathbb{N}^{*}\right) .
$$

Therefore, we see from (3.29) and (3.30) that, in order to obtain (3.6), it suffices to estimate the sequence $\left(C_{m}\right)_{m \geq 1}$. This will be done by distinguishing two cases.

First case. Assume that $\mu_{m}$ is a term of the sequence $\left(\mu_{k}^{(1)}\right)$, which has been defined in (3.11). We deduce from (3.10) and from (3.28) that $C_{m}^{2}=2 / 3$. This fact, combined with (3.29) implies that (3.6) holds in the considered case.

Second case. Assume that $\mu_{m}$ is not a term of the sequence $\left(\mu_{k}^{(1)}\right)$. In this case, we know from (3.12) that $D_{m}=2 C_{m} \cos \mu_{m}$. Using this information in (3.28) it follows that

$$
C_{m}^{2}=\left[1-\frac{\sin 4 \mu_{m}}{4 \mu_{m}}+2 \cos ^{2}\left(\mu_{m}\right)-\cos ^{2}\left(\mu_{m}\right) \frac{\sin 2 \mu_{m}}{\mu_{m}}+M \sin ^{2}\left(2 \mu_{m}\right)\right]^{-1} .
$$

On the other hand, by using (3.14), (3.15) and (3.16), we deduce

$$
\begin{gathered}
\sin ^{2}\left(2 \mu_{m}^{(i)}\right) \rightarrow 0, \quad \frac{\sin 4 \mu_{m}^{(i)}}{4 \mu_{m}^{(i)}} \rightarrow 0, \quad \cos ^{2}\left(\mu_{m}^{(i)}\right) \frac{\sin 2 \mu_{m}^{(i)}}{\mu_{m}^{(i)}} \rightarrow 0 \quad(i \in\{2,3\}) \\
1+2 \cos ^{2}\left(2 \mu_{m}^{(2)}\right) \rightarrow 1, \quad 1+2 \cos ^{2}\left(2 \mu_{m}^{(3)}\right) \rightarrow 3
\end{gathered}
$$

Gathering (3.31) and (3.32) yields

$$
\left|C_{m}\right| \geqslant c_{1} \quad\left(m \in \mathbb{N}^{*}\right)
$$

for some constant $c_{1}>0$.

We conclude that (3.6) holds for the two cases and the proof is now complete.

Combining Propositions 2.2, 3.1 and 3.2, we conclude

Proposition 3.3. The pair $(A, B)$ is null-controllable in any time $\tau>0$ with control cost $K(\tau)<M_{0} \mathrm{e}^{\frac{M_{1}}{\tau}}$ for some positive constants $M_{0}$ and $M_{1}$.

Now we turn to the controllability of the system (2.33) in the particular case where $A, B$ are defined by (3.1) and (3.3) and $C: X \rightarrow \mathbb{R}$ is defined by

$$
C\left[\begin{array}{l}
\varphi \\
g
\end{array}\right]=g \quad\left(\left[\begin{array}{l}
\varphi \\
g
\end{array}\right] \in X\right)
$$

i.e. we consider the controllability of the nonhomogeneous system

$$
\left\{\begin{array}{l}
\dot{z}=A z+B u+f, \\
\dot{h}=C z \\
z(0)=z_{0}, \\
h(0)=h_{0} .
\end{array}\right.
$$


Proposition 3.4. With the above notation for $A, B$ and $C$, let $\tau>0$ and let $M_{0}, M_{1}$ be the constants in Proposition 3.3. Let $\rho_{\mathcal{F}}, \rho_{0}:[0, \tau) \rightarrow[0, \infty)$

$$
\left\{\begin{array}{l}
\rho_{\mathcal{F}}(t)=\exp \left(-\frac{\alpha}{(\tau-t)^{2}}\right), \\
\rho_{0}(t)=M_{0} \exp \left(\frac{M_{1}}{(q-1)(\tau-t)}-\frac{\alpha}{q^{4}(\tau-t)^{2}}\right),
\end{array}\right.
$$

where the parameters $q, \alpha$ satisfy:

$$
q>1, \quad \alpha>\frac{M_{1} q^{4}}{2(q-1)} \tau .
$$

Let $(\mathcal{F}, \mathcal{U}, \mathcal{Z})$ be the spaces obtained from $\rho_{\mathcal{F}}$ and $\rho_{0}$ according to (2.13). Then there exists a bounded linear operator

$$
E_{\tau}: X \times \mathbb{R} \times \mathcal{F} \rightarrow \mathcal{U}
$$

such that for any $\left(z_{0}, h_{0}, f\right) \in X \times \mathbb{R} \times \mathcal{F}$, the control $u=E_{\tau}\left(z_{0}, h_{0}, f\right)$ is such that the solution of (3.35) satisfies $z \in \mathcal{Z}$ and $h(\tau)=0$.

Proof. We consider the adjoint system (see (2.34)) of (3.35)

$$
\left\{\begin{array}{l}
-\dot{\xi}(t)=A \xi(t)+g(t)+C^{*} r, \\
\xi(\tau)=0
\end{array}\right.
$$

where $g:[0, \infty) \rightarrow X$ and $r \in \mathbb{R}$. It is not difficult to check that our assumptions (3.37) imply that the functions defined in (3.36) satisfy (2.10)-(2.12). This fact, combined with Proposition 2.3 (with $\gamma(t)=M_{0} \mathrm{e}^{\frac{M_{1}}{t}}$ ) and Corollary 2.6 yields that there exists a $C>0$ with

$$
\|\xi(0)\|_{X}^{2}+\int_{0}^{\tau}\left\|\rho_{\mathcal{F}} \xi\right\|_{X}^{2} \mathrm{~d} t \leq C\left(\int_{0}^{\tau}\left\|\rho_{0}\left(g+C^{*} r\right)\right\|_{X}^{2} \mathrm{~d} t+\int_{0}^{\tau}\left\|\rho_{0} B^{*} \xi\right\|_{U}^{2} \mathrm{~d} t\right),
$$

for every solution of (3.39). In order to eliminate $r$ from the right-hand side of the above inequality, we show that

$$
|r|^{2} \leq C\left(\int_{0}^{\tau}\left\|\rho_{0} g\right\|_{X}^{2} \mathrm{~d} t+\int_{0}^{\tau}\left\|\rho_{0} B^{*} \xi\right\|_{U}^{2} \mathrm{~d} t\right)
$$

for every solution of (3.39). This is done by employing a compactness-uniqueness argument. Assume that (3.41) is false, then there exists a sequence $\left(g_{n}, r_{n}\right) \in L^{2}([0, \tau], X) \times \mathbb{R}$ such that $\left|r_{n}\right|^{2}=1$ for every $n \geqslant 1$ and

$$
\int_{0}^{\tau}\left\|\rho_{0} g_{n}\right\|_{X}^{2} \mathrm{~d} t+\int_{0}^{\tau}\left\|\rho_{0} B^{*} \xi_{n}\right\|_{U}^{2} \mathrm{~d} t \rightarrow 0
$$

where $\xi_{n}, g_{n}$ and $r_{n}$ satisfy

$$
\left\{\begin{array}{l}
-\dot{\xi}_{n}(t)=A \xi_{n}(t)+g_{n}(t)+C^{*} r_{n}, \\
\xi_{n}(\tau)=0 .
\end{array}\right.
$$

By compactness we deduce that, up to the extraction of a subsequence,

$$
r_{n} \rightarrow r, \quad|r|=1
$$

Moreover, (3.42) implies that

$$
\rho_{0} g_{n} \rightarrow 0 \quad \text { strongly in } \quad L^{2}([0, \tau], X)
$$

Let

$$
\rho_{1}(t)=M_{0} \exp \left(\frac{M_{1}}{(q-1)(\tau-t)}-\frac{2 \alpha}{(\tau-t)^{2}}\right) \quad(t \in(0, \tau))
$$


It can be easily verified that

$$
\rho_{1}(t) \leqslant \rho_{0}(t) \quad t \in[0, \tau]
$$

so that, using (3.45), we obtain that

$$
\rho_{1} g_{n} \rightarrow 0 \quad \text { strongly in } \quad L^{2}([0, \tau], X) .
$$

On the other hand, it is not difficult to check that there exists a $C_{1}>0$ with

$$
\left|\dot{\rho}_{1}(t)\right| \leq C_{1} \rho_{\mathcal{F}}(t) \quad t \in[0, \tau] .
$$

This last estimate, combined with (3.40) and (3.42), implies that

$$
\left\|\dot{\rho}_{1} \xi_{n}\right\|_{L^{2}([0, \tau], X)} \leq C_{1}\left\|\rho_{\mathcal{F}} \xi_{n}\right\|_{L^{2}([0, \tau], X)} \leq C \quad(n \geqslant 1),
$$

for some positive constant $C$. Multiplying both sides of (3.43) by $\rho_{1}(t)$, we deduce that

$$
\left\{\begin{array}{l}
-\left(\rho_{1} \xi_{n}\right)_{t}=A\left(\rho_{1} \xi_{n}(t)\right)+\rho_{1} g_{n}(t)+\rho_{1} C^{*} r_{n}-\dot{\rho}_{1} \xi_{n}(t), \\
\xi_{n}(\tau)=0
\end{array}\right.
$$

Let $f_{n}=\rho_{1} g_{n}+\rho_{1} C^{*} r_{n}-\dot{\rho}_{1} \xi_{n}$. Combining (3.44), (3.46) and (3.47) implies that $\left(f_{n}\right)$ is bounded in $L^{2}([0, \tau], X)$. Hence, using Lemma 2.5 for (3.48), we deduce that $\left(\rho_{1} \xi_{n}\right)$ is bounded in $L^{2}([0, \tau], \mathcal{D}(A)) \cap H^{1}((0, \tau), X)$. Since $\rho_{1}$ is bounded away from zero on $[0, \tau-\epsilon]$ for every $\epsilon>0$, we have

$$
\xi_{n} \rightarrow \xi \quad \text { weakly in } \quad L^{2}([0, \tau-\epsilon], \mathcal{D}(A)) \cap H^{1}((0, \tau-\epsilon), X),
$$

up to the extraction of a subsequence. The function $\xi$ above obviously satisfies the first equation (3.39) for $t \in[0, \tau-\epsilon]$. Therefore denoting $\xi=\left[\begin{array}{l}\phi \\ k\end{array}\right]$, after passing to the limit in (3.43), we have

$$
\begin{gathered}
\xi \in L^{2}([0, \tau-\epsilon], \mathcal{D}(A)) \cap H^{1}((0, \tau-\epsilon), X), \\
\left\{\begin{array}{l}
-\phi_{t}(t, x)=\phi_{x x}(t, x), \quad(0, \tau-\epsilon) \times\{(-2,0) \cup(0,1)\} \\
-M \dot{k}(t)=\left[\phi_{x}\right](t, 0)+r, \\
k(t)=\phi(t, 0), \\
\phi(t,-2)=0, \\
\phi(t, 1)=0 .
\end{array}\right.
\end{gathered}
$$

Recalling the relation (3.42), the definition (3.36) of $\rho_{0}$ and the formula (3.4) for $B^{*}$, we see that for every $\epsilon>0, \phi=0$ in $(0, \tau-\epsilon) \times(-2,-3 / 2)$. Using a unique continuation property for the heat equation (see, for instance, [12], Thm. 1.1), we deduce that $\phi=0$ on $(0, \tau-\epsilon) \times(-2,0)$. Recalling the definition of $A$ and $\mathcal{D}(A)$ from (3.1) together with (3.49), we see that

$$
\phi(t, 0+)=\phi(t, 0-)=0,
$$

for almost every $t \in(0, \tau-\epsilon)$, so that $\phi$ satisfies the heat equation with homogeneous Dirichlet boundary conditions for $t \in(0, \tau-\epsilon)$ and $x \in(0,1)$. It follows that $\phi$ can be written

$$
\phi(t, x)=\sum_{n=1}^{\infty} a_{n} \sin (n \pi x) \mathrm{e}^{-\pi^{2} n^{2}(\tau-t-\epsilon)} \quad(t \in[0, \tau-\epsilon], \quad x \in(0,1)),
$$

for some real sequence $\left(a_{n}\right)$ with $\left(n a_{n}\right) \in l^{2}$. 
On the other hand, using the third equation in (3.50) and (3.51), it follows that $k(t)=0$ for every $t \in[0, \tau-\epsilon)$. By using the second equation of (3.50) and the fact that $\phi$ vanishes for $x<0$, yields

$$
\phi_{x}(t, 0+)=-r \quad(t \in[0, \tau-\epsilon]) .
$$

The above equation and (3.52) give

$$
r+\pi \sum_{n=1}^{\infty} n a_{n} \mathrm{e}^{-\pi^{2} n^{2}(\tau-t-\epsilon)}=0 \quad(t \in[0, \tau-\epsilon]),
$$

which clearly implies that $a_{n}=0$ for every $n \geqslant 1$ and that $r=0$. The last assertion contradicts (3.44). We can thus conclude that (3.41) holds.

Using next (3.41) in (3.40) it follows that there exists a positive constant $C$ such that

$$
|r|^{2}+\|\xi(0)\|^{2}+\int_{0}^{\tau}\left\|\rho_{\mathcal{F}} \xi\right\|^{2} \mathrm{~d} t \leq C\left(\int_{0}^{\tau}\left\|\rho_{0} g\right\|^{2} \mathrm{~d} t+\int_{0}^{\tau}\left\|\rho_{0} B^{*} \xi\right\|_{U}^{2} \mathrm{~d} t\right)
$$

for every solution of (3.39). By applying Proposition 2.9 (with $Y=\mathbb{R}$ ) we obtain the desired result.

Corollary 3.5. With the assumptions and notation in Proposition 3.4, denote

$$
\rho(t)=\mathrm{e}^{-\frac{\beta}{(\tau-t)^{2}}} \quad(t \in[0, \tau)),
$$

where the positive constant $\beta$ is such that $\beta<\frac{\alpha}{q^{4}}$. Then, for every $z_{0} \in \mathcal{D}\left((-A)^{1 / 2}\right), f \in \mathcal{F}$ and $h_{0} \in \mathbb{R}$, the trajectory $z$ obtained by solving (3.35) with $u=E_{\tau}\left(z_{0}, h_{0}, f\right)$ is in $C\left([0, \tau], \mathcal{D}\left((-A)^{1 / 2}\right)\right)$. Moreover, there exists a positive constant $C$ such that

$$
\left\|\frac{z}{\rho}\right\|_{C\left([0, \tau] ; \mathcal{D}\left((-A)^{1 / 2}\right)\right) \cap H^{1}((0, \tau), X) \cap L^{2}([0, \tau], \mathcal{D}(A))} \leq C\left(\left\|z_{0}\right\|_{\mathcal{D}\left((-A)^{1 / 2}\right)}+\left|h_{0}\right|+\left\|\frac{f}{\rho_{\mathcal{F}}}\right\|_{L^{2}([0, \tau], X)}\right),
$$

for every $z_{0} \in \mathcal{D}\left((-A)^{1 / 2}\right), f \in \mathcal{F}$ and $h_{0} \in \mathbb{R}$. Finally, if the parameter $q$ from the definition (3.36) of $\rho_{0}$ satisfies $q^{4}<2$ then

$$
\frac{\rho^{2}}{\rho_{\mathcal{F}}} \in C[0, \tau]
$$

Proof. Since $\beta<\frac{\alpha}{q^{4}}$, we see that the functions $\rho_{0}, \rho_{\mathcal{F}}$ and $\rho$ satisfy (2.30). Therefore (3.54) follows by applying Proposition 2.8 .

Furthermore, if we choose $q$ such that $1<q^{4}<2$, then $\frac{\rho^{2}}{\rho_{\mathcal{F}}} \in C[0, \tau]$ since

$$
\frac{\rho^{2}}{\rho_{\mathcal{F}}}(t)=\exp \left(\frac{\alpha-2 \beta}{(\tau-t)^{2}}\right)
$$

is bounded on $[0, \tau]$ if $\alpha-2 \beta<0$. Using again the fact that $\beta<\frac{\alpha}{q^{4}}$, we deduce that we can achieve this by choosing such a $\beta$ iff $q^{4}<2$.

\section{Proof of the main Result}

This section is devoted to the proof of Theorem 1.1. Using a standard methodology for parabolic control problems we first study, instead of the boundary control problem (1.1), a distributed control problem (see (4.1) below). To deal with this system with moving boundary, we first use a change of variable to transform it (see (4.4) below). This system can be written as system (3.35) but with a right-hand side $f$ depending on $z$ 
and $h$. Lemma 4.1 gives properties of this nonlinear term and thus using a fixed-point argument and the results of the previous section, we deduce the null-controllability of (4.4) for small initial data. Using our change of variables, it implies the null-controllability of (4.4) for small initial data of the distributed control problem (4.1) (Thm. 4.3). We end this section by proving that the controllability of (4.1) implies the controllability of (1.1), which proves Theorem 1.1.

First, let us consider a distributed control problem associated to (1.1):

$$
\begin{cases}v_{t}(t, x)-v_{x x}(t, x)+v(t, x) v_{x}(t, x)=\hat{u}(t, x) & (t \geqslant 0, \quad x \in[-2,1] \backslash\{h(t)\}), \\ v(t, h(t))=\dot{h}(t) & (t \geqslant 0), \\ M \ddot{h}(t)=\left[v_{x}\right](t, h(t)) & (t \geqslant 0), \\ v(t,-2)=0, \quad v(t, 1)=0 & (t \geqslant 0), \\ h(0)=h_{0}, \quad \quad \dot{h}(0)=h_{1}, & \\ v(0, x)=v_{0}(x) \quad x \in[-2,1] \backslash\left\{h_{0}\right\}, & \end{cases}
$$

where $\hat{u}(t, x)$ is the input function, supported in the subinterval $(-2,-5 / 4)$, and the state of the system is the triple $\left[\begin{array}{l}v \\ h \\ h\end{array}\right]$. The study of the above internal controllability problem should be seen only as a tool in the proof of our main boundary controllability result. Therefore, we choose here the very particular interval $[-2,1]$ for technical purposes (as already explained in the previous section), but this will be enough to obtain the proof of our main result.

To study the control problem (4.1) we reduce it to a problem in a fixed domain by using an appropriate change of variables. More precisely, let $\tau>0$ and let $h:[0, \tau] \rightarrow(-1,1)$ be a function in $H^{1}(0, \tau)$. For every $t \in[0, \tau]$ we define a diffeomorphism $\eta(t, \cdot)$ from $(-2, h(t)) \cup(h(t), 1)$ onto $(-2,0) \cup(0,1)$ by

$$
y=\eta(t, x)= \begin{cases}\frac{x-h(t)}{1-h(t)}, & x>h(t) \\ \frac{2(x-h(t))}{2+h(t)}, & x<h(t) .\end{cases}
$$

We define a new function $\varphi$ by setting

$$
\varphi(t, \eta(t, x))=v(t, x) \quad(t \in[0, \tau], x \in(-2, h(t)) \cup(h(t), 1)) .
$$

It is not difficult to check that, using the change of variables defined by (4.2) and (4.3), the system (4.1) becomes

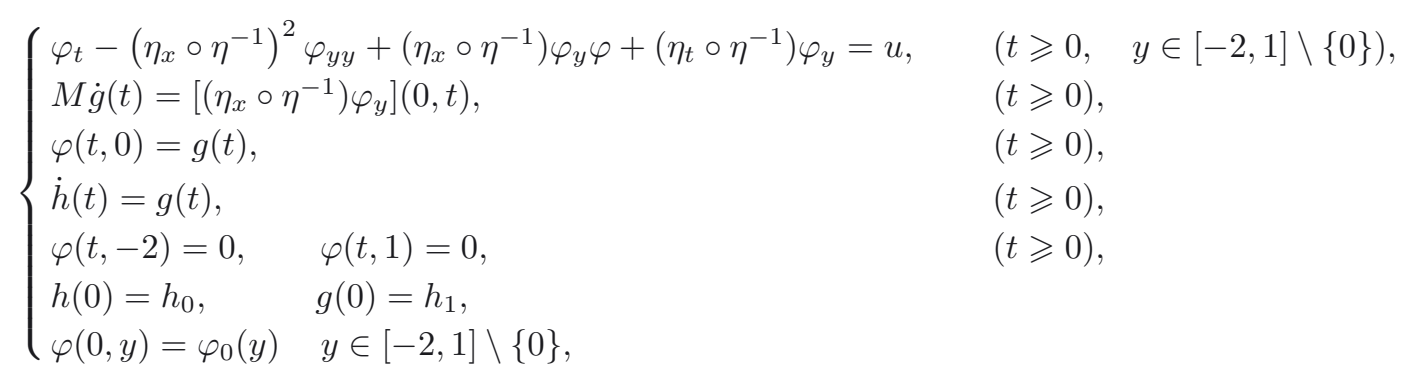

where $u$ is a new input function and

$$
\left[\left(\eta_{x} \circ \eta^{-1}\right) \varphi_{y}\right](t, 0)=\left[\left(\eta_{x} \circ \eta^{-1}\right)\right](t, 0) \varphi_{y}(t, 0+)+\left(\eta_{x} \circ \eta^{-1}\right)(t, 0-)\left[\varphi_{y}\right](t, 0) .
$$


We note that, since the function $\eta$ is given by (4.2), we have

$$
\begin{gathered}
\left(\eta_{x} \circ \eta^{-1}\right)(t, y)=\frac{1}{1-\kappa(y) h(t)}= \begin{cases}\frac{1}{1-h(t)}, & y>0, \\
\frac{2}{2+h(t)}, & y<0 .\end{cases} \\
\left(\eta_{t} \circ \eta^{-1}\right)(t, y)=-g(t) \frac{1-\kappa(y) y}{1-\kappa(y) h(t)}= \begin{cases}-\frac{1-y}{1-h(t)} g(t) & \text { for } \quad y>0, \\
-\frac{2+y}{2+h(t)} g(t) & \text { for } \quad y<0 .\end{cases}
\end{gathered}
$$

where

$$
\kappa(y)=\left\{\begin{array}{l}
1 \quad \text { for } \quad y>0 \\
-\frac{1}{2} \quad \text { for } \quad y<0
\end{array}\right.
$$

Moreover, we have

$$
\left[\eta_{x} \varphi_{y}\right](t, 0)=\left(\frac{1}{1-h(t)}-\frac{2}{2+h(t)}\right) \varphi_{y}(t, 0+)+\frac{2}{2+h(t)}\left[\varphi_{y}\right](t, 0) .
$$

Using the above formula, combined with (4.5) and (4.6), we obtain that (4.4) writes in the more explicit form

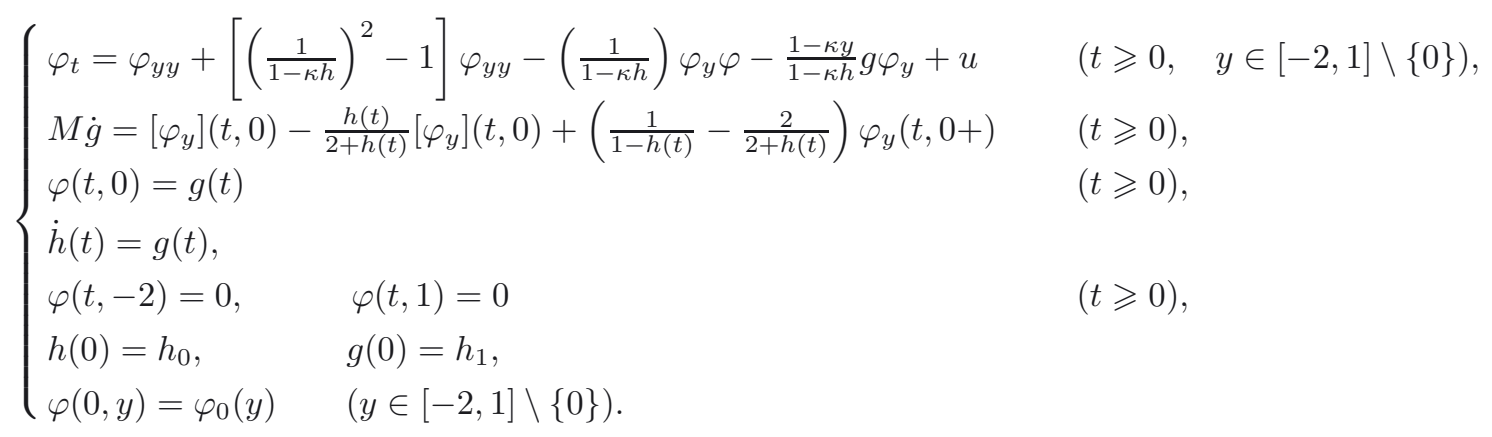

The above system suggests the introduction of the nonlinear operator

$$
N: \mathcal{D}(A) \times(-2,1) \rightarrow X
$$

defined by

$$
N\left[\begin{array}{c}
z \\
h
\end{array}\right]=\left[\begin{array}{c}
{\left[\left(\frac{1}{1-\kappa h}\right)^{2}-1\right] \varphi_{y y}-\left(\frac{1}{1-\kappa h}\right) \varphi_{y} \varphi-\frac{1-\kappa y}{1-\kappa h} g \varphi_{y}} \\
-\frac{h}{2+h}\left[\varphi_{y}\right](0)+\left(\frac{1}{1-h}-\frac{2}{2+h}\right) \varphi_{y}(0+)
\end{array}\right]
$$

where $z=\left[\begin{array}{l}\varphi \\ g\end{array}\right]$ is a generic element of $\mathcal{D}(A)$. Lemma below yields some important properties of $N$.

Lemma 4.1. For every $a \in(0,1)$, there exists a positive constant $C=C(a)$ such that

$$
\begin{array}{r}
\left\|N\left[\begin{array}{c}
z \\
h
\end{array}\right]\right\|_{X} \leq C\left(|g|^{2}+\|\varphi\|_{H^{1}}^{2}+|h|\left\|\varphi_{y y}\right\|_{L^{2}}\right) \quad\left(\left[\begin{array}{l}
z \\
h
\end{array}\right] \in \mathcal{D}(A) \times(-a, a)\right) \\
\left\|N\left[\begin{array}{c}
z_{1} \\
h_{1}
\end{array}\right]-N\left[\begin{array}{c}
z_{2} \\
h_{2}
\end{array}\right]\right\|_{X} \leq C\left(\left(\left|h_{1}-h_{2}\right|+\left|g_{1}-g_{2}\right|\right)\left(\left\|\varphi_{1, y y}\right\|_{L^{2}}+\left\|\varphi_{1}\right\|_{H^{1}}^{2}+\left\|\varphi_{2}\right\|_{H^{1}}\right)\right. \\
\left.+\left|h_{2}\right|\left\|\varphi_{1}-\varphi_{2}\right\|_{H^{2}}+\left\|\varphi_{1}^{2}-\varphi_{2}^{2}\right\|_{H^{1}}\right) \quad\left(\left[\begin{array}{c}
z_{i} \\
h_{i}
\end{array}\right] \in \mathcal{D}(A) \times(-a, a), i=1,2\right) .
\end{array}
$$


Proof. The proof of (4.9) follows easily from standard embedding and trace results for Sobolev spaces.

To prove (4.10), we note that

$$
N=\left[\begin{array}{c}
F_{1}+F_{2}+F_{3} \\
F_{4}
\end{array}\right],
$$

where

$$
\begin{gathered}
F_{1}\left[\begin{array}{l}
z \\
h
\end{array}\right]=\left[\left(\frac{1}{1-\kappa h}\right)^{2}-1\right] \varphi_{y y}, \quad F_{2}\left[\begin{array}{l}
z \\
h
\end{array}\right]=-\left(\frac{1}{1-\kappa h}\right) \varphi_{y} \varphi \\
F_{3}\left[\begin{array}{c}
z \\
h
\end{array}\right]=-\frac{1-\kappa y}{1-\kappa h} g \varphi_{y} \\
F_{4}\left[\begin{array}{l}
z \\
h
\end{array}\right]=-\frac{h}{2+h}\left[\varphi_{y}\right](0)+\left(\frac{1}{1-h}-\frac{2}{2+h}\right) \varphi_{y}(0+) .
\end{gathered}
$$

Simple calculations, combined with standard embedding and trace results for Sobolev spaces, imply that there exists a constant $C>0$, depending only on $a$, such that for every $\left[\begin{array}{c}z_{1} \\ h_{1}\end{array}\right],\left[\begin{array}{l}z_{2} \\ h_{2}\end{array}\right] \in \mathcal{D}(A) \times(-a, a)$ we have

$$
\begin{gathered}
\left\|F_{1}\left[\begin{array}{l}
z_{1} \\
h_{1}
\end{array}\right]-F_{1}\left[\begin{array}{c}
z_{2} \\
h_{2}
\end{array}\right]\right\|_{L^{2}} \leqslant C\left(\left|h_{1}-h_{2}\right|\left\|\varphi_{1, y y}\right\|_{L^{2}}+\left|h_{2}\right|\left\|\varphi_{1, y y}-\varphi_{2, y y}\right\|_{L^{2}}\right), \\
\left\|F_{2}\left[\begin{array}{c}
z_{1} \\
h_{1}
\end{array}\right]-F_{2}\left[\begin{array}{c}
z_{2} \\
h_{2}
\end{array}\right]\right\|_{L^{2}} \leqslant C\left(\left|h_{1}-h_{2}\right|\left\|\varphi_{1}\right\|_{H^{1}}^{2}+\left\|\varphi_{1}^{2}-\varphi_{2}^{2}\right\|_{H^{1}}\right), \\
\left\|F_{3}\left[\begin{array}{c}
z_{1} \\
h_{1}
\end{array}\right]-F_{3}\left[\begin{array}{c}
z_{2} \\
h_{2}
\end{array}\right]\right\|_{L^{2}} \leqslant C\left(\left|h_{1}-h_{2}\right|\left|g_{1}\right|\left\|\varphi_{1}\right\|_{H^{1}}+\left|g_{1}\right|\left\|\varphi_{1}-\varphi_{2}\right\|_{H^{1}}+\left|g_{1}-g_{2}\right|\left\|\varphi_{2}\right\|_{H^{1}}\right), \\
\left|F_{4}\left[\begin{array}{c}
z_{1} \\
h_{1}
\end{array}\right]-F_{4}\left[\begin{array}{c}
z_{2} \\
h_{2}
\end{array}\right]\right| \leqslant C\left(\left|h_{1}-h_{2}\right|\left\|\varphi_{1, y y}\right\|_{L^{2}}+\left|h_{2}\right|\left\|\varphi_{1, y y}-\varphi_{2, y y}\right\|_{L^{2}}\right) .
\end{gathered}
$$

The above estimates clearly imply the conclusion.

The main step in the proof of Theorem 1.1 is the following result.

Proposition 4.2. Let $\tau>0$. Then there exists $r>0$ such that for every $h_{0}, h_{1}, \varphi_{0}$ satisfying

$$
\begin{gathered}
\left|h_{0}\right|+\left|h_{1}\right|+\left\|\varphi_{0}\right\|_{H_{0}^{1}(-2,1)} \leqslant r, \\
\varphi_{0}(0)=h_{1} .
\end{gathered}
$$

The nonlinear system (4.8) is null controllable in time $\tau$ with a control u supported in $(-2,-3 / 2)$ : there exists $u \in L^{2}([0, \tau] \times[-2,-3 / 2])$ such that the corresponding solution satisfies

$$
\varphi(\tau, \cdot)=0, \quad h(\tau)=0, \quad \dot{h}(\tau)=0 .
$$

Moreover, the control $u$ can be chosen so that

$$
-1<h(t)<1 \quad(t \in[0, \tau]) .
$$


Proof. Since we look for controls such that $u(t, \cdot)$ is supported in $(-2,-3 / 2)$, the problem can be rewritten

$$
\left\{\begin{array}{l}
\dot{z}=A z+N\left[\begin{array}{l}
z \\
h
\end{array}\right]+B u, \\
\dot{h}=C z \\
z(0)=z_{0}, \\
h(0)=h_{0},
\end{array}\right.
$$

with the same choice of the operators $A, B$ and $C$ as in Section 3 (see (3.1), (3.3) and (3.34)).

In order to prove the null controllability of (4.22) we consider the linearized problem

$$
\left\{\begin{array}{l}
\dot{z}=A z+B u+f, \\
\dot{h}=C z, \\
z(0)=z_{0}, \\
h(0)=h_{0},
\end{array}\right.
$$

with $f:[0, \tau] \rightarrow X$. Let $\rho_{\mathcal{F}}, \rho_{0}, \rho$ be the functions introduced in Proposition 3.4 and in Corollary 3.5 and let $(\mathcal{F}, \mathcal{U}, \mathcal{Z})$ be the corresponding function spaces, defined according to (2.13). According to Proposition 3.4 and Corollary 3.5 there exists a control $u=E_{\tau}\left(z_{0}, h_{0}, f\right)$ such that the corresponding trajectory $(z, h)$ satisfies $h(\tau)=0$ together with (3.54). It follows that, denoting

$$
\mathcal{F}_{r}=\left\{f \in \mathcal{F} \mid\|f\|_{\mathcal{F}} \leq r\right\},
$$

with $r>0$ small enough, we can define an operator $\mathcal{N}$ acting on $\mathcal{F}_{r}$ by

$$
\mathcal{N}(f)(t):=N\left[\begin{array}{l}
z(t) \\
h(t)
\end{array}\right] \quad(t \in[0, \tau]),
$$

where $\left[\begin{array}{l}z \\ h\end{array}\right]$ is the state trajectory of (4.23) corresponding to the input $u=E_{\tau}\left(z_{0}, h_{0}, f\right)$. Indeed, the fact that, provided that $r$ is small enough, we have $h(t) \in(-1,1)$ for every $t \in[0, \tau]$ (so that $\mathcal{N}$ is well-defined on $\mathcal{F}_{r}$ ) follows from Corollary 3.5. More precisely, let $\rho$ the function defined in (3.53). Since $\rho(\tau)=h(\tau)=0$ and $\dot{\rho}(t)=-\frac{\beta}{(\tau-t)^{3}} \rho(t)$, by the Cauchy mean value theorem, for every $t \in[0, \tau]$ we have

$$
\left|\frac{h(t)}{\rho(t)}\right|=\left|\frac{h(t)-h(\tau)}{\rho(t)-\rho(\tau)}\right| \leqslant\left\|\frac{g}{\dot{\rho}}\right\|_{C[0, \tau]} \leq C\left\|\frac{g}{\rho}\right\|_{C[0, \tau]},
$$

where $C$ is a constant depending only on $\beta$ and on $\tau$. By (3.54), if we choose $r$ small enough, we have (4.21), so that $\mathcal{N}$ is indeed well defined.

In order to obtain the conclusion of the proposition, it suffices to check that, for $r>0$ small enough, $\mathcal{N}$ is contractive mapping from $\mathcal{F}_{r}$ into itself. We first check that $\mathcal{F}_{r}$ is invariant for $\mathcal{N}$, provided that $r$ is small enough. This follows from Lemma 4.1, noting that, for almost every $t \in[0, \tau]$, we have

$$
\left\|\frac{\mathcal{N}(f)}{\rho_{\mathcal{F}}}(t)\right\|_{X} \leq C\left|\frac{\rho^{2}(t)}{\rho_{\mathcal{F}}(t)}\right|\left(\left|\frac{g(t)}{\rho(t)}\right|^{2}+\left|\frac{h(t)}{\rho(t)}\right|\left\|\frac{\varphi(t, \cdot)}{\rho(t)}\right\|_{H^{2}}+\left\|\frac{\varphi(t, \cdot)}{\rho(t)}\right\|_{H^{1}}^{2}\right) .
$$

Since $\rho$ and $\frac{\rho^{2}}{\rho_{\mathcal{F}}}$ are continuous functions on $[0, \tau]$ (see (3.36) and (3.55)), it follows that

$$
\|\mathcal{N}(f)\|_{\mathcal{F}} \leq C\left(\left\|\frac{g}{\rho}\right\|_{C[0, \tau]}^{2}+\left\|\frac{h}{\rho}\right\|_{C[0, \tau]}\left\|\frac{\varphi}{\rho}\right\|_{L^{2}\left([0, \tau], H^{2}\right)}+\left\|\frac{\varphi}{\rho}\right\|_{C\left([0, \tau], H^{1}\right)}^{2}\right) .
$$


Using (3.54), (4.19), (4.25) and (4.26), it follows that

$$
\|\mathcal{N}(f)\|_{\mathcal{F}} \leq C r^{2} \quad\left(f \in \mathcal{F}_{r}\right),
$$

for some positive constant $C$. The last estimate implies indeed that $\mathcal{N}$ invariates $\mathcal{F}_{r}$ for $r>0$ small enough.

We still have to show that $\mathcal{N}$ is contracting for $r$ small enough. Using (4.10) it follows that there exists $C>0$, independent of $r>0$, such that the inequality

$$
\begin{aligned}
\frac{1}{\rho_{\mathcal{F}}}\left\|\mathcal{N}\left(f_{1}\right)-\mathcal{N}\left(f_{2}\right)\right\|_{X} \leq & \frac{C \rho^{2}}{\rho_{\mathcal{F}}}\left(\frac{\left|h_{1}-h_{2}\right|}{\rho}+\frac{\left|g_{1}-g_{2}\right|}{\rho}\right)\left(\left\|\frac{\varphi_{1, y y}}{\rho}\right\|_{L^{2}}+\rho\left\|\frac{\varphi_{1}}{\rho}\right\|_{H^{1}}^{2}+\left\|\frac{\varphi_{2}}{\rho}\right\|_{H^{1}}\right) \\
& +\frac{C \rho^{2}}{\rho_{\mathcal{F}}} \frac{\left|h_{2}\right|+\left|g_{1}\right|}{\rho}\left(\left\|\frac{\mid \varphi_{1, y y}-\varphi_{2, y y}}{\rho}\right\|_{L^{2}}+\rho\left\|\frac{\varphi_{1}^{2}-\varphi_{2}^{2}}{\rho^{2}}\right\|_{H^{1}}\right) \quad\left(f_{1}, f_{2} \in \mathcal{F}_{r}\right),
\end{aligned}
$$

holds for $t \in[0, \tau]$. Integrating the last estimate on $[0, \tau]$ and using (4.25) it follows that there exists $r^{*}>0$ and a constant $C>0$, not depending on $r \in\left(0, r^{*}\right)$, such that

$$
\left\|\mathcal{N}\left(f_{1}\right)-\mathcal{N}\left(f_{2}\right)\right\|_{\mathcal{F}} \leq C r\left(\left\|\frac{g_{1}-g_{2}}{\rho}\right\|_{C[0, \tau]}+\left\|\frac{\varphi_{1, y y}-\varphi_{2, y y}}{\rho}\right\|_{L^{2}\left([0, \tau], L^{2}\right)}+\left\|\frac{\varphi_{1}-\varphi_{2}}{\rho}\right\|_{C\left([0, \tau], H^{1}\right)}\right) .
$$

Since $z=z_{1}-z_{2}, h=h_{1}-h_{2}$ satisfy (3.35), with $z_{0}=0, h_{0}=0$ and $f=f_{1}-f_{2}$, we can use (3.54) to obtain that $\mathcal{N}$ is indeed contracting on $\mathcal{F}_{r}$, provided that $r$ is small enough.

As a consequence of the last proposition we obtain the following result:

Theorem 4.3. The system (4.1) is locally null controllable in any time $\tau>0$. More precisely, for every $\tau>0$ there exists $r>0$ such that every initial data $h_{0}, h_{1}, v_{0}$ satisfying

$$
\left|h_{0}\right|+\left|h_{1}\right|+\left\|v_{0}\right\|_{H_{0}^{1}(-2,1)} \leq r, \quad v_{0}\left(h_{0}\right)=h_{1},
$$

can be steered to rest by means of a control, $\hat{u} \in L^{2}\left([0, \tau], L^{2}(-2,-5 / 4)\right)$. Moreover, $\widehat{u}$ can be chosen such that $h \in C[0, \tau]$ and

$$
-1<h(t)<1 \quad(t \in[0, \tau]) .
$$

Proof. According to Proposition 4.2, we can find $u \in L^{2}\left(0, \tau ; L^{2}(-2,-3 / 2)\right)$ such that the solution of (4.8) satisfies $h \in C[0, \tau]$, together with (4.29) and

$$
\varphi(\tau, \cdot)=0, \quad g(\tau)=h(\tau)=0 .
$$

Using the change of variables (4.2), we obtain a controlled trajectory of the system (4.1), which is vanishing for $t=\tau$ and with a control $\widehat{u}$ such that

$$
\operatorname{supp} \widehat{u}(t, \cdot) \subset(-2, \hat{a}) \quad(t \in[0, \tau]) .
$$

It can be seen by (4.2) that

$$
\frac{2(\hat{a}-h(t))}{2+h(t)}=\eta(t, \hat{a}) \leq-\frac{3}{2} .
$$

This, combined with (4.29) implies that $\hat{a} \leq-\frac{5}{4}$, which finishes the proof.

We are now in a position to prove our main result. 
Proof of Theorem 1.1. Since $v_{0} \in H_{0}^{1}(-1,1)$, we can extend it by zero on $(-2,-1)$ such that $v_{0} \in H_{0}^{1}(-2,1)$. We consider system (4.1) with initial data $\left(v_{0}, h_{0}, h_{1}\right)$. Then, there exists a constant $r>0$ such that if

$$
\left|h_{0}\right|+\left|h_{1}\right|+\left\|v_{0}\right\|_{H_{0}^{1}\left((-2,1) \backslash\left\{h_{0}\right\}\right)} \leq r,
$$

there exists a control

$$
\hat{u} \in L^{2}\left([0, \tau], L^{2}(-2,-5 / 4)\right)
$$

such that the solution of (4.1) satisfies (4.29) and

$$
\begin{gathered}
v \in L^{2}\left([0, \tau], H^{2}(-2,1)\right) \cap C\left([0, \tau], H^{1}(-2,1)\right) \cap H^{1}\left((0, \tau), L^{2}(-2,1)\right), \\
v(\tau, \cdot)=0, \quad h(\tau)=\dot{h}(\tau)=0 .
\end{gathered}
$$

Define

$$
u(t)=v(-1, t) \quad t \in[0, \tau] .
$$

We clearly have $u \in C[0, \tau]$ and $u$ drives the system (1.1) to rest in time $\tau$.

\section{REFERENCES}

[1] A. Bensoussan, G. Da Prato, M.C. Delfour and S.K. Mitter, Representation and control of infinite-dimensional systems, Systems 8 Control: Foundations $\&$ Applications 1. Birkhäuser Boston Inc., Boston, MA (1992).

[2] M. Boulakia and A. Osses, Local null controllability of a two-dimensional fluid-structure interaction problem. ESAIM: COCV 14 (2008) 1-42.

[3] S. Dolecki and D.L. Russell, A general theory of observation and control. SIAM J. Control Optim. 15 (1977) 185-220.

[4] A. Doubova and E. Fernández-Cara, Some control results for simplified one-dimensional models of fluid-solid interaction. Math. Models Methods Appl. Sci. 15 (2005) 783-824.

[5] H.O. Fattorini and D.L. Russell, Exact controllability theorems for linear parabolic equations in one space dimension. Arch. Rational Mech. Anal. 43 (1971) 272-292.

[6] A.V. Fursikov and O.Yu. Imanuvilov, Controllability of Evolution Equations, Lecture Notes Series 34. Seoul National University Research Institute of Mathematics, Global Analysis Research Center, Seoul (1996).

[7] F. Gozzi and P. Loreti. Regularity of the minimum time function and minimum energy problems: the linear case. SIAM J. Control Optim. 37 (1999) 1195-1221 (electronic).

[8] O. Imanuvilov and T. Takahashi, Exact controllability of a fluid-rigid body system. J. Math. Pures Appl. (9) 87 (2007) $408-437$.

[9] O.Y. Imanuvilov, Remarks on exact controllability for the Navier-Stokes equations. ESAIM: COCV 6 (2001) 39-72 (electronic).

[10] G. Lebeau and L. Robbiano, Contrôle exact de l'équation de la chaleur. Comm. Partial Differential Equations 20 (1995) $335-356$.

[11] L. Miller, A direct Lebeau-Robbiano strategy for the observability of heat-like semigroups. Discrete Contin. Dyn. Syst., Ser. B 14 (2010) 1465-1485.

[12] J.-C. Saut and B. Scheurer, Unique continuation for some evolution equations. J. Differential Equations 66 (1987) $118-139$.

[13] G. Tenenbaum and M. Tucsnak, On the null-controllability of diffusion equations. ESAIM: COCV 17 (2011) 1088-1100.

[14] G. Tenenbaum and M. Tucsnak, New blow-up rates for fast controls of Schrödinger and heat equations. J. Differential Equations 243 (2007) 70-100.

[15] M. Tucsnak and G. Weiss, Observation and control for operator semigroups. Birkhäuser Advanced Texts: Basler Lehrbücher [Birkhäuser Advanced Texts: Basel Textbooks], Birkhäuser Verlag, Basel (2009).

[16] J.L. Vázquez and E. Zuazua, Large time behavior for a simplified 1D model of fluid-solid interaction. Comm. Partial Differential Equations 28 (2003) 1705-1738.

[17] J.L. Vázquez and E. Zuazua, Lack of collision in a simplified 1D model for fluid-solid interaction. Math. Models Methods Appl. Sci. 16 (2006) 637-678. 